\title{
Multiple Ocular Diseases Detection by Graph Regularized Multi-label Learning
}

\author{
Xiangyu Chen, Yanwu Xu, Lixin Duan, Zhuo Zhang, \\ Damon Wing Kee Wong, and Jiang Liu \\ Institute for Infocomm Research, Agency for Science, Technology and Research, Singapore
}

\begin{abstract}
We develop a general framework for multiple ocular diseases diagnosis, based on Graph Regularized Multi-label Learning (GRML). Glaucoma, Pathological Myopia (PM), and Age-related Macular Degeneration (AMD) are three leading ocular diseases in the world. By exploiting the correlations among these three diseases, a novel GRML scheme is investigated for a simultaneous detection of these three leading ocular diseases for a given fundus image. We validate our GRML framework by conducting extensive experiments on SiMES dataset. The results show area under curve (AUC) of the receiver operating characteristic curve in multiple ocular diseases detection are much better than traditional popular algorithms. The method could be used for glaucoma, PM, and AMD diagnosis.
\end{abstract}

\section{Introduction}

Glaucoma, Pathological Myopia (PM), and age-related macular degeneration (AMD) are three leading ocular diseases. Glaucoma is a chronic eye disease that leads to vision loss, in which the optic nerve is progressively damaged. It is one of the common causes of blindness, and is predicted to affect around 80 million people by 2020 [1].

Glaucoma diagnosis is typically based on the medical history, intraocular pressure, and visual field loss tests together with a manual assessment of the Optic Disc (OD) through ophthalmoscopy. OD or optic nerve head is the location where ganglion cell axons exit the eye to form the optic nerve [21][20], through which visual information of the photo-receptors is transmitted to the brain [2].

As one of the leading causes of blindness worldwide, Pathological Myopia (PM) is a type of severe and progressive nearsightedness characterized by changes in the fundus of the eye, due to posterior staphyloma and deficient corrected acuity. PM is primarily a genetic condition [3] [4]. It is accompanied by degenerative changes in the retina, which if left untreated can lead to irrecoverable vision loss. The accurate detection of PM will enable timely intervention and facilitate better disease management to slow down the progression of the disease. PM has been detected mostly from fundus image where retinal degeneration is observed in the form of Peripapillary Atrophy (PPA). PPA is the thinning of retinal layers around the optic nerve and is characterized by a pigmented ring like structure around the optic disc.

Age-related Macular Degeneration (AMD) causes vision loss at the central region and blur and distortion at the peripheral region. Depending on the presence of exu- 

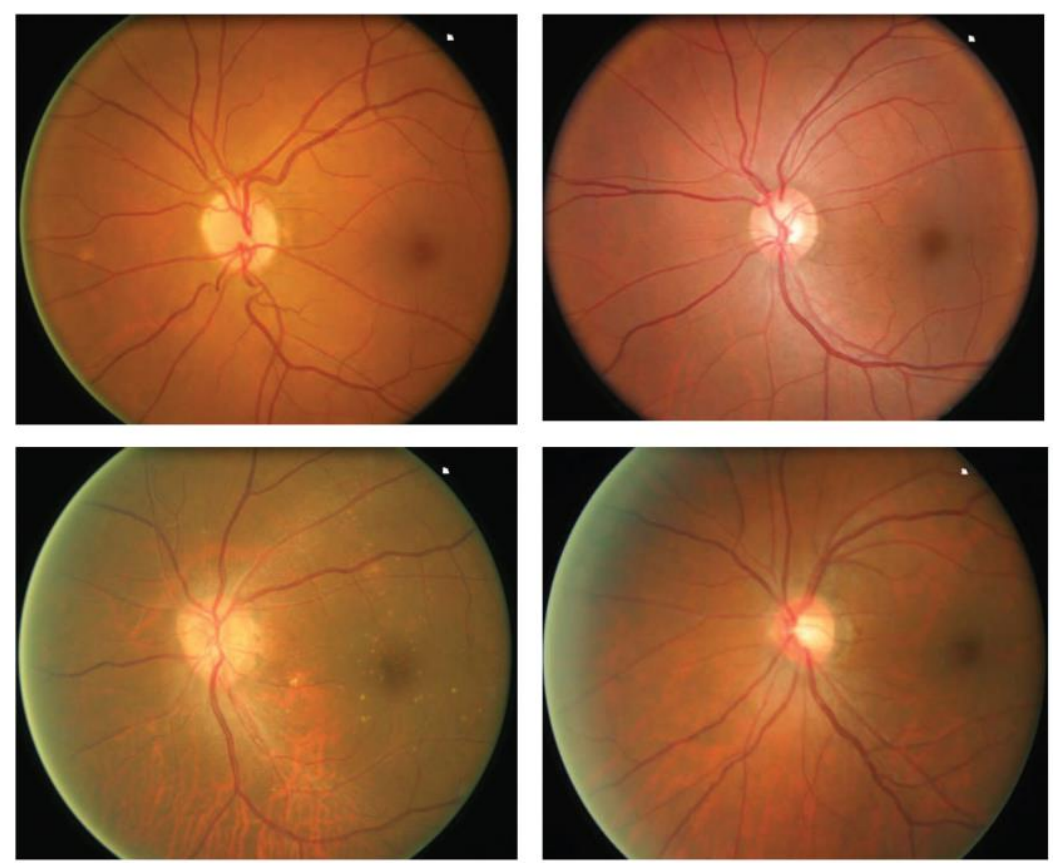

Fig. 1. Three leading ocular diseases (from left top to right bottom): a) Normal eye, b) Glaucoma disease, c) Age-related Macular Degeneration (AMD) disease, d) Pathological Myopia (PM) disease.

dates, AMD is classified into dry AMD (non-exudative AMD) and wet AMD (exudative AMD). Dry AMD results from atrophy of the retinal pigment epithelial layer below the retina. It causes vision loss through loss of photoreceptors (rods and cones) in the central part of the retina. The major symptom and also the first clinical indicator of dry AMD is drusen, sub-retinal deposits formed by retinal waste. Wet AMD causes vision loss due to abnormal blood vessel growth (choroidal neovascularization) in the choriocapillaris, through Bruchs membrane, ultimately leading to blood and protein leakage below the macula. Bleeding, leaking, and scarring from these blood vessels eventually cause irreversible damage to the photoreceptors and rapid vision loss if left untreated. The major symptom of wet AMD is exudation [5] [6].

For these three leading ocular diseases, there are some correlations among them. In recent decades the problem of low vision and blindness in elderly people became major and socially significant issue. The number of patients having age-related macular degeneration (AMD) in association with glaucoma grows all over the world [7], which attaches medical and social value to this multiple diseases diagnosis problem. Moreover, in recent study, myopic eyes are less likely to have AMD and diabetic retinopathy (DR) but more likely to have nuclear cataract and primary open-angle glaucoma (POAG) [8]. Inspired by the correlations among Glaucoma, PM, and AMD, we propose a graph regularized multi-label learning framework for harmoniously integrating the above correlation information, and investigating the problem of learn- 
ing to simultaneously diagnose these three leading ocular diseases for a given fundus image. Unlike previous approaches that detect individual ocular disease independently, our proposed GRML scheme encodes the correlation information of multiple diseases of an image as a unit label confidence vector, which naturally imposes interlabel constraints and manipulates labels of diseases interactively. Since KullbackLeibler divergence has been widely employed in the field of computer vision [9] [14][19], here we adopt the probabilistic Kullback-Leibler divergence for problem formulation on multi-label learning. This kind of multiple diseases screening is more oriented to real world diagnosis scenario because a patient may have two or three diseases at the same time.

\section{Graph Regularized Multi-label Learning}

There are two main concatenating parts in our proposed multi-label learning framework: 1) Graph Construction based on k-nearest-neighbor (k-NN); 2) Multi-label Learning based on Kullback-Leibler divergence.

\subsection{Graph Construction}

In this paper, we adopt an directed weighted graph $G=\langle V, E\rangle$ for multi-label learning, where $G$ is represented by a weight matrix $W=\left\{w_{i j}\right\} \in \square^{m \times m}$, the cardinality of the node set is $m=l+u$ ( $l$ and $u$ denote the number of labeled and unlabeled fundus images respectively), and the edge set $E \subseteq V \times V$ describes the graph topology. We denote the sets of labeled and unlabeled vertices $V_{l}$ and $V_{u}$ respectively. To enforce the constructed graph to be sparse, we utilize the k-nearest-neighbor (k-NN) for graph construction. The assumption of k-NN is that the weight between two nodes $w_{i j}$ is nonzero only when $j \in N_{i}$, where $N_{i}$ denotes the local neighborhood of the i-th image.

The graph construction includes two parts: neighborhood selection and weight computation. For neighborhood selection, we employ k-nearest-neighbor for graph construction. In this setting, if $x_{j}$ is among the k-nearest neighbors to the $\mathrm{i}$-th fundus image, then $w_{i j}$ is nonzero. Graphs constructed in this way ensure a constant vertex degree and avoid over-dense sub-graphs and isolated vertices. For weight computation, the underlying philosophy is that the message transmitted from the neighboring vertices with higher weights will be much stronger than the others, where the more similar a sample is to another sample, the larger weight exists between them. In this paper, we utilize the popular way to calculate the pairwise weights-exponentially weighted similarity. The weights between the neighbors are computed as below:

$$
w_{i j}=\exp \left(-\frac{d_{G}\left(x_{i}, x_{j}\right)}{\sigma^{2}}\right),
$$

where $d_{G}\left(x_{i}, x_{j}\right)$ is the distance between the fundus image $i$ and $j$, and $\sigma$ is a free parameter to control the decay rate. 


\subsection{Multi-label Learning based on Kullback-Leibler Divergence}

Here we adopt the same representation and distance measure as in [14], yet in a different scenario (i.e. medical multiple diseases detection). We denote the set of labeled fundus images by $M_{l}=\left\{x_{i}, r_{i}\right\}_{i=1}^{l}$, where $x_{i}$ is the feature vector of the $\mathrm{i}$-th fundus image and $r_{i}$ is a multi-label vector with ground truth. $M_{u}=\left\{x_{i}\right\}_{i=l+1}^{l+u}$ is the set of unlabeled images, and $M=\left\{M_{l}, M_{u}\right\}$ is the entire fundus image data set. Given $x_{i}, p_{i}$ be the probability measure over the measurable space $(Y, \Psi)$, where $\Psi$ is the $\sigma$-field of measurable subsets of $Y$ and $Y \subset .^{\cdot}$ is the space of classifier outputs. For binary classification, $|Y|=2$. For multiple diseases classification, $|Y|>2$, which is the case of our proposed framework. We use $p_{i}$ and $r_{i}$ for the i-th fundus image, both of which are subject to the multinomial distributions, and $p_{i}(y)$ is the probability that xi belongs to class $y$. Here $\left\{r_{j}, j \in V_{l}\right\}$ encodes the supervision disease information of the labeled fundus image. We adopt the following objective function for multi-label learning, which is based on the concept of Kullback-Leibler divergence:

$$
Z_{1}(p)=\sum_{l=1}^{l} Z_{K L}\left(r_{i} \| p_{i}\right)+\mu \sum_{i=1}^{m} \sum_{j \in N(i)} w_{i j} Z_{K L}\left(p_{i} \| p_{j}\right)
$$

Here $Z_{K L}\left(r_{i} \| p_{i}\right)$ denotes the KL divergence between $r_{i}$ and $p_{i}$, whose formal definition for the discrete case is expressed as $Z_{K L}\left(r_{i}|| p_{i}\right)=\sum_{y} r_{i}(y) \log \left(r_{i}(y) / p_{i}(y)\right)$.

Since $Z_{1}$ in Equation (2) is not amenable to alternating optimization, we employ a modified version by introducing a new group of variables $\left\{q_{i}\right\}$, which is shown as below:

$$
Z_{1}(p)=\sum_{l=1}^{l} Z_{K L}\left(r_{i} \| q_{i}\right)+\mu \sum_{i=1}^{m} \sum_{j \in N(i)} w_{i j} Z_{K L}\left(p_{i} \| p_{j}\right)+\eta \sum_{i=1}^{m} Z_{K L}\left(p_{i} \| q_{i}\right)
$$

where $q_{i}$ can actually be regarded as an approximated version of $p_{i}$, which is introduced to decouple the original term $\mu \sum_{i=1}^{m} \sum_{j \in N(i)} w_{i j} Z_{K L}\left(p_{i} \| p_{j}\right)$. To enforce consistency between them, the third term $\eta \sum_{i=1}^{m} Z_{K L}\left(p_{i} \| q_{i}\right)$ is incorporated. The Equation (3) could be solved by utilizing the similar method in [14].

\section{Experiments}

To evaluate the multiple diseases diagnosis performance of our proposed Graph Regularized Multi-label Learning (GRML), we conduct extensive experiments on the Singapore Malay Eye Study (SiMES) database [10] for detecting the three leading ocular diseases: Glaucoma, Pathological Myopia (PM), and age-related macular degeneration (AMD). We also consider using different types of features as well as their combination in the experiments, which gives us a total of three settings: 1) global features; 2) bag of words; 3) global feature + bag of words. The notation + indicates a combination of two types of features in the corresponding setting. 


\subsection{Dataset}

SiMES is a population-based study conducted from 2004 to 2007. It examined a crosssectional and age stratified sample of 3,280 randomly selected Malays aged from 40 to 80 years old living in Singapore. For each subject in this database, personal demographic/ clinical data, a retinal fundus image, and a blood sample (used for genotyping) were collected during the clinic visit, which thus gives us three informatics domains containing completely different types of data. Moreover, the detection of three leading ocular diseases (i.e., glaucoma, AMD, and PM) have been made by clinicians at the same time. The detection of different diseases made by clinicians during the visit are used as the gold standard to evaluate the classification performance of all the methods in this work. In this work, we select a subset of SiMES for experiments, which contains 2,258 subjects. Among the 2,258 subjects, there are 100 with glaucoma, 122 with AMD, and 58 with PM. For each disease, the distribution of the subjects who contracted the disease in the selected dataset is representative of the disease prevalence in the population.

\subsection{Low-level Features}

To facilitate experimentation and comparison of results, we extract a set of effective and popularly used global and local features for each image. For global features, four types of features are extracted: 64-dimensional color histogram (LAB) [11], 144dimensional color auto-correlogram (HSV) [12], 73-dimensional edge direction histogram [13], and 128-dimensional wavelet texture [15]. For local features, 500dimensional bags of visual words [16] are generated. The generation of bag of words comprises three major steps: (a) we apply the Difference of Gaussian filter on the gray scale images to detect a set of key-points and scales respectively; (b) we compute the Scale Invariant Feature Transform (SIFT) [16] over the local region defined by the key-point and scale; and (c) we perform the vector quantization on SIFT region descriptors to construct the visual vocabulary by exploiting the k-means clustering.

\subsection{Evaluation Metric}

In this work, we utilize the area under the curve (AUC) of receiver operation characteristic curve (ROC) to evaluate the performance of glaucoma diagnosis. The ROC is plotted as a curve which shows the tradeoff between sensitivity TPR (true positive rate) and specificity TNR (true negative rate), defined as

$$
T P R=\frac{T P}{T P+F N}, \quad T N R=\frac{T N}{T N+F P}
$$

where TP and TN are the number of true positives and true negatives, respectively, and FP and FN are the number of false positives and false negatives, respectively. 
Table 1. The AUCs of different algorithms for simultaneously detecting the three leading ocular diseases (i.e., Glaucoma, PM and AMD) on SiMES dataset. The combined visual features (global features + bag of words) are utilized in the experiment. The results of AUC marked in boldfaceare significantly better than others.

\begin{tabular}{|c|c|c|c|}
\hline Methods & Glaucoma & PM & AMD \\
\hline KNN & $74.2 \%$ & $86.5 \%$ & $72.9 \%$ \\
\hline SVM & $76.7 \%$ & $89.1 \%$ & $75.0 \%$ \\
\hline Our Proposed & $\mathbf{8 2 . 5 \%}$ & $\mathbf{9 2 . 3 \%}$ & $\mathbf{7 9 . 3 \%}$ \\
\hline
\end{tabular}

\subsection{Experimental Setup}

In this work, we compare our proposed Graph Regularized Multi-label Learning (GRML) with two baseline methods (i.e., Support Vector Machine (SVM) [17] and the k- Nearest Neighbors (KNN) [18]) under the aforementioned three settings using different feature types as well as their combinations. For each setting, all the methods for the automatic detections of the three leading ocular diseases (i.e., glaucoma, AMD and PM) are evaluated on the subset of SiMES.

\subsection{Experiment Results Analysis}

In the experiments, we systematically compare our proposed GRML with SVM and KNN on the subset of SiMES under different types of visual features. Below are the parameters and the adopted values for each method:

- For SVM algorithm, we adopt the RBF kernel. For its two parameters $\gamma$ and $C$, we set $\gamma=0.6$ and $C=1$ in experiments after fine tuning.

- For KNN, there is only one parameter $k$ for tuning, which stands for the number of nearest neighbors and is trivially set as 500 .

- For GRML, we set the two parameters as $\eta=5$, and $\mu=10$.

The AUCs of these three methods for simultaneously detecting the three leading ocular diseases (i.e., Glaucoma, PM, and AMD) on SiMES dataset are illustrated in Table 1. The combined visual features (global features + bag of words) are utilized in this experiment. Our proposed algorithm GRML outperforms the other baseline algorithms significantly. For example, GRML has an improvement $7.6 \%$ over SVM, $11.2 \%$ over KNN for detecting Glaucoma. For PM, GRML has an improvement $3.6 \%$ over SVM, $6.7 \%$ over KNN. For AMD, GRML has an improvement 5.7\% over SVM, $8.8 \%$ over KNN. The improvement is supposed to stem from the fact that our proposed algorithm encodes the disease label information of each image as a unit confidence vector, which imposes extra inter-label constraints. In contrast, other methods consider each disease label independently.

The comparison results of the detecting performance under three feature setting are listed in Table 2, Table 3, and Table 4. From Table 2, we are able to observe that, for glaucoma detection, our proposed algorithm GRML outperforms the two baseline 
Table 2. The AUCs of different algorithms under three setting of features on SiMES dataset for Glaucoma diagnosis. The AUCs marked in boldface are significantly better than others.

\begin{tabular}{|c|c|c|c|}
\hline Methods & KNN & SVM & Our Proposed \\
\hline Global Features & $71.2 \%$ & $73.5 \%$ & $78.7 \%$ \\
\hline Bag of Words & $68.4 \%$ & $70.9 \%$ & $75.0 \%$ \\
\hline Combined Features & $\mathbf{7 4 . 2 \%}$ & $\mathbf{7 6 . 7 \%}$ & $\mathbf{8 2 . 5 \%}$ \\
\hline
\end{tabular}

Table 3. The AUCs of different algorithms under three setting of features on SiMES dataset for AMD diagnosis. The AUCs marked in boldface are significantly better than others.

\begin{tabular}{|c|c|c|c|}
\hline Methods & KNN & SVM & Our Proposed \\
\hline Global Features & $70.2 \%$ & $72.5 \%$ & $76.4 \%$ \\
\hline Bag of Words & $68.1 \%$ & $70.3 \%$ & $73.5 \%$ \\
\hline Combined Features & $\mathbf{7 2 . 9 \%}$ & $\mathbf{7 5 . 0 \%}$ & $\mathbf{7 9 . 3 \%}$ \\
\hline
\end{tabular}

Table 4. The AUCs of different algorithms under three setting of features on SiMES dataset for PM diagnosis. The AUCs marked in boldface are significantly better than others.

\begin{tabular}{|c|c|c|c|}
\hline Methods & KNN & SVM & Our Proposed \\
\hline Global Features & $81.5 \%$ & $84.1 \%$ & $87.3 \%$ \\
\hline Bag of Words & $83.8 \%$ & $86.5 \%$ & $89.5 \%$ \\
\hline Combined Features & $\mathbf{8 6 . 5 \%}$ & $\mathbf{8 9 . 1 \%}$ & $\mathbf{9 2 . 3 \%}$ \\
\hline
\end{tabular}

algorithms based on the combined features. The AUC of the receiver operating characteristic curve in glaucoma detection is $82.5 \%$. The similar results are shown in Table 3 and Table 4 for AMD and PM detection respectively. For AMD detection, our proposed GRML algorithm achieves $79.3 \%$. For PM detection, the AUC of GRML is $92.3 \%$.

\section{Conclusions}

In this paper, we present a novel Graph Regularized Multi-label Learning (GRML) framework for multiple ocular diseases diagnosis. The proposed GRML harmoniously integrates the correlation information of Glaucoma, PM and AMD, and exploits the problem of learning to simultaneously detect these three ocular diseases. Two concatenating parts are included GRML: 1) Graph Construction based on k-nearest-neighbor (k-NN); 2) Multi-label Learning based on Kullback-Leibler Divergence. This type of multiple diseases screening is more oriented to real world diagnosis scenario because a patient may has two or three diseases at the same time. In future work, we plan to extend our multi-label learning framework to large-scale multiple ocular diseases diagnosis.

\section{References}

1. Quigley, H.A., Broman, A.T.: The number of people with glaucoma worldwide in 2010 and 2020. Br. J. Ophthalmol, 90(3), 262-7, (2006) 
2. Xu, Y., Lin, S., Wong, T.Y., Liu, J., Xu, D.: Efficient Reconstruction-Based Optic Cup Localization for Glaucoma Screening. In: MICCAI 2013

3. Young, T.L., Ronan, S.M.,Alvear, A.B., Wildenberg, S.C., Oetting, W.S., Atwood, L.D., Wilkin, D.J., King, R.A.: A second locus for familial high myopia maps to chromosome 12q. Am J Hum Genet., 63(5), 1419-24 (1998)

4. Xu, Yanwu, Liu, Jiang, Zhang, Zhuo, Tan, Ngan Meng,Wong, DamonWing Kee, Saw, Seang Mei, Wong, Tien Yin: Learn to recognize pathological myopia in fundus images using bag-of-feature and sparse learning approach. In: International Symposium on Biomedical Imaging (2013)

5. Bressler, N.M., Bressler, S.B., Fine, S.L.: Age-related macular degeneration. Survey of ophthalmology. Survey of ophthalmology, 32(6), 375-413 (1988)

6. De Jong, P.T.: Age-related macular degeneration. : New England Journal of Medicine, 355(14), 1474-1484 (2006)

7. Avetisov, S.E., Erichev, V.P., Budzinskaia, M.V., Karpilova, M.A., Gurova, I.V., Shchegoleva, I.V., Chikun, E.A.: Age-related macular degeneration and glaucoma: intraocular pressure monitoring after intravitreal injections. : Vestn. Oftalmol. , 128(6), 3-5, (2012)

8. Pan, C.W., Cheung, C.Y., Aung, T., Cheung, C.M., Zheng, Y.F., Wu, R.Y., Mitchell, P., Lavanya, R, Baskaran, M., Wang, J.J., Wong, T.Y., Saw, S.M.: Differential associations of myopia with major age-related eye diseases: the Singapore Indian Eye Study. Ophthalmol, 20(2), 284-91, (2013)

9. Subramanya, A., Bilmes, J.: Entropic graph regularization in non-parametric semisupervised classification. In: NIPS, (2009)

10. Shen, S. Y., Wong, T. Y., Foster, P. J., Loo, J. L., Rosman, M., Loon, S. C., Wong, W. L., Saw, S. M., Aung, T.: The prevalence and types of glaucoma in malay people: the Singapore malay eye study. Investigative Ophthalmology and Visual Science, 49(9), 38463851, (2008)

11. Shapiro, L. G., Stockman, G. C.: Computer Vision. : Prentice Hall, (2003)

12. Huang, J., Kumar, S., Mitra, M., Zhu, W.-J., Zabih, R.: Image indexing using color correlogram. In: IEEE Conf. on Computer Vision and Pattern Recognition, (1997)

13. Park, D. K., Jeon, Y. S., Won, C. S.: Efficient use of local edge histogram descriptor. In: ACM Multimedia, (2000)

14. Chen, X., Mu, Y., Yan, S., Chua, T.: Efficient Large-Scale Image Annotation by Probabilistic Collaborative Multi-Label Propagation. In: ACM Multimedia, (2010)

15. Manjunath, B. S., Ma, W.-Y.: Texture features for browsing and retrieval of image data. IEEE Transactions on Pattern Analysis and Machine Intelligence, 18(8), 837-842, (1996)

16. Lowe., D.: Distinctive image features from scale-invariant keypoints. Intl J. Computer Vision, 2(60), 91-110, (2004)

17. Collobert, R., Sinz, F. H., Weston, J., Bottou, L.: Large scale transductive svms. : Journal of Machine Learning Research, 7, 1687-1712, (2006)

18. Duda, R., Stork, D., Hart, P.: Pattern Classification. JOHN WILEY, (2000)

19. Chen, X., Mu, Y., Liu, H., Yan, S., Rui, Y., Chua, T.: Large-scale multilabel propagation based on efficient sparse graph construction. ACM Transactions on Multimedia Computing, Communications and Applications, 10(1), 6, (2013)

20. Xu, Y., Liu, J., Lin, S., Xu, D., Cheung, C., Aung, T., Wong, T.Y.: Efficient Optic Cup Detection from Intra-image Learning with Retinal Structure Priors. In: MICCAI 2012

21. Xu, Y., Xu, D., Lin, S., Liu, J., Cheng, J., Cheung, C., Aung, T.,Wong, T.Y.: Sliding Window and Regression Based Cup Detection in Digital Fundus Images for Glaucoma Diagnosis. In: MICCAI 2011 